\title{
Struktur von flüssigem Quecksilber
}

\author{
H. Richter und G. Breitling \\ Max-Planck-Institut für Metallforschung, Stuttgart \\ und Medizinisches Strahleninstitut der Universität Tübingen \\ (Z. Naturforschg. 22 a, 658-662 [1967]; eingegangen am 19. Januar 1967)
}

\begin{abstract}
Flüssiges Hg wurde bei Zimmertemperatur mit Röntgen-Strahlen untersucht. Wie bei geschmolzenem $\mathrm{Bi}$ wird die Struktur von flüssigem $\mathrm{Hg}$ von zwei Komponenten bestimmt, und zwar von der dichtesten Kugelpackung mit $r_{1}=3,04 \AA$ als kürzestem Atomabstand und von der FlächengitterStruktur mit $r_{1}{ }^{\prime}=2,86 \AA$ als kürzestem Atomabstand. Bei $\mathrm{Hg}$ ist bereits das vierte Maximum der $4 \pi r^{2}\left[\varrho(r)-\varrho_{0}\right]$-Kurve in ein Maximum der Flächengitter-Struktur und in ein Maximum der dichtesten Kugelpackung aufgespalten. Danach kommen in flüssigem $\mathrm{Hg}$ die gerade Atomkette der dichtesten Kugelpackung und die gerade Atomkette der Flächengitter-Struktur nebeneinander vor. Weiter zeigt die Aufteilung der Maxima in einen Beitrag der dichtesten Kugelpackung und in einen solchen der Flächengitter-Struktur, daß für Atomabstände $r \leqq 2 r_{1}$ die dichteste Kugelpackung, für Atomabstände $r \geqq 3 r_{1}{ }^{\prime}$ dagegen die Flächengitter-Struktur dominierend ist.

Die Doppelstruktur der Metallschmelze zeichnet sich in der $4 \pi r^{2}\left[\varrho(r)-\varrho_{0}\right]$-Kurve von flüssigem $\mathrm{Hg}$ wie bei geschmolzenem $\mathrm{Bi}$ erst bei großen Abstandswerten unmittelbar ab, hier allerdings sehr eindrucksvoll. In beiden Fällen liegen gerade Atomketten vor; nur wenige Atomabstände innerhalb dieser Ketten legen den charakteristischen Verlauf der Intensitäts- und der Atomverteilungskurve fest.
\end{abstract}

Die Erfahrungen, welche bei der Diskussion der $4 \pi r^{2}\left[Q(r)-\varrho_{0}\right]$-Kurven von geschmolzenem $\mathrm{Bi}$ gemacht wurden, sind sicherlich bei flüssigem $\mathrm{Hg}$ von Nutzen. In Abb. $1 \mathrm{a}$ ist die $4 \pi r^{2}\left[\varrho(r)-\varrho_{0}\right]$-Kurve von flüssigem $\mathrm{Hg}$ wiedergegeben; sie wurde durch Fourier-Analyse der $i(s)$ exp.-Kurve erhalten, wie sie Pfannenschmid ${ }^{1}$ mit Röntgen-Strahlen aufgenommen hat. Zur Deutung der Maximalagen nimmt man an, daß in flüssigem $\mathrm{Hg}$ wie in geschmolzenem $\mathrm{Bi}$ zweierlei gerade Atomketten mit den nur wenig voneinander verschiedenen kürzesten Atomabständen $r_{1}$ und $r_{1}{ }^{\prime}$ vorkommen. So bezeichnen die einfachen Pfeile $\downarrow$ die Atomabstände $r_{v}=v r_{1}$ innerhalb der geraden Atomkette der dichtesten Kugelpackung mit $r_{1}=3,04 \AA$ als kürzestem Atomabstand, und die Pfeile mit Querbalken $\downarrow$ markieren die Atomabstände $r_{v}{ }^{\prime}=v r_{1}{ }^{\prime}$ innerhalb der geraden Atomkette der Flächengitter-Struktur mit $r_{1}^{\prime}=2,86 \AA$ als kürzestem Atomabstand. Das erste Maximum tritt beim Atomabstande $r_{1}=3,03 \AA$ auf, also etwa beim kürzesten Atomabstande der dichtesten Kugelpackung (vgl. Tab. 1). Das zweite Maximum bei $r=5,87 \AA$ kommt durch Überlagerung der Maxima bei den doppelten Atomabständen $2 r_{1}{ }^{\prime}=2 \cdot 2,86 \AA=5,72 \AA$

1 O. Pfannenschmid, Dissertation, Technische Hochschule Stuttgart 1959; Z. Naturforschg. 15a, 603 [1960].

2 Nach Tab. 1, Spalte 4, ist im Mittel $r=11,44 \AA=4 r_{1}{ }^{\prime}$ oder $r_{1}{ }^{\prime}=2,86 \AA$ als kürzester Atomabstand der FlächengitterStruktur. Bei der abschnittweisen Fourier-Analyse der Intensitätskurve von flüssigem $\mathrm{Hg}$ fanden Breitling, HandtManx und Richter ${ }^{3}$ dagegen $r_{1}{ }^{\prime}=2,98 \AA$. Wegen der niedrigen Untersuchungstemperatur von $18^{\circ} \mathrm{C}$ ist bei $\mathrm{Hg}$ der und $2 r_{1}=2 \cdot 3,04 \AA=6,08 \AA$ der FlächengitterStruktur und der dichtesten Kugelpackung zustande. Die dreifachen Atomabstände $3 r_{1}{ }^{\prime}=3 \cdot 2,86 \AA=$ $8,58 \AA$ und $3 r_{1}=3 \cdot 3,04 \AA=9,12 \AA$ zeichnen sich im dritten Maximum gesondert ab, obwohl die Trennung beider Strukturen erstmals im vierten Maximum, d.h. durch die Maxima bei ${ }^{2} r=11,47 \AA$ $\approx 4 r_{1}{ }^{\prime}=4 \cdot 2,86 \AA=11,44 \AA$ und bei $r=12,11 \AA$ $\approx 4 r_{1}=4 \cdot 3,04 \AA=12,16 \AA$, vollkommen ist. In Abb. 1 b bildet sich sogar die fünfte Maximumgruppe beider Strukturen bei $r=14,38 \AA \approx 5 r_{1}{ }^{\prime}$ $=5 \cdot 2,86 \AA=14,30 \AA$ und bei $r=15,14 \AA \approx 5 r_{1}$ $=5 \cdot 3,04 \AA=15,20 \AA$ noch deutlich ab.

Die $i(s)_{\text {exp. }}$-Kurve von flüssigem $\mathrm{Hg}$ wurde bei verschiedenen Integrationslängen $s_{2}$ analysiert. So sind in den Abb. $1 \mathrm{~b}$ und $\mathrm{c}$ zwei weitere $4 . \pi r^{2}$ $\cdot\left[\varrho(r)-\varrho_{0}\right]$-Kurven für $s_{2} \lessgtr 12,6$ dargestellt. Erst für $s_{2}=13,5$ hebt sich in Abb. $1 \mathrm{c}$ auf der abfallenden Flanke des dritten Maximums der FlächengitterStruktur deutlich sichtbar das dritte Maximum der dichtesten Kugelpackung ab. Die experimentellen Lagen der Maxima sind in Tab. 1 zusammengestellt. Man sieht, daß die Maximalagen der einzelnen Spalten trotz verschiedener Integrationslängen $s_{2}$ prak-

kürzeste Atomabstand $r_{1}=3,04 \AA$ der dichtesten Kugelpackung noch an der Fixierung des hinteren Teiles der Intensitätskurve beteiligt; das trifft aber für die Schmelzen von $\mathrm{Au}, \mathrm{Ag}, \mathrm{Pb}, \mathrm{Tl}$ u. a. m. nicht zu. Die bei $\mathrm{Au}, \mathrm{Ag} \ldots$ erhaltenen Verhältniswerte für die beobachteten Atomabstände $r_{1} / r_{1}{ }^{\prime} \approx 1,07$ sind ein Kriterium dafür.

3 G. Breitling, D. Handtmann u. H. Richter, Z. Phys. 178, 294 [1964]. 


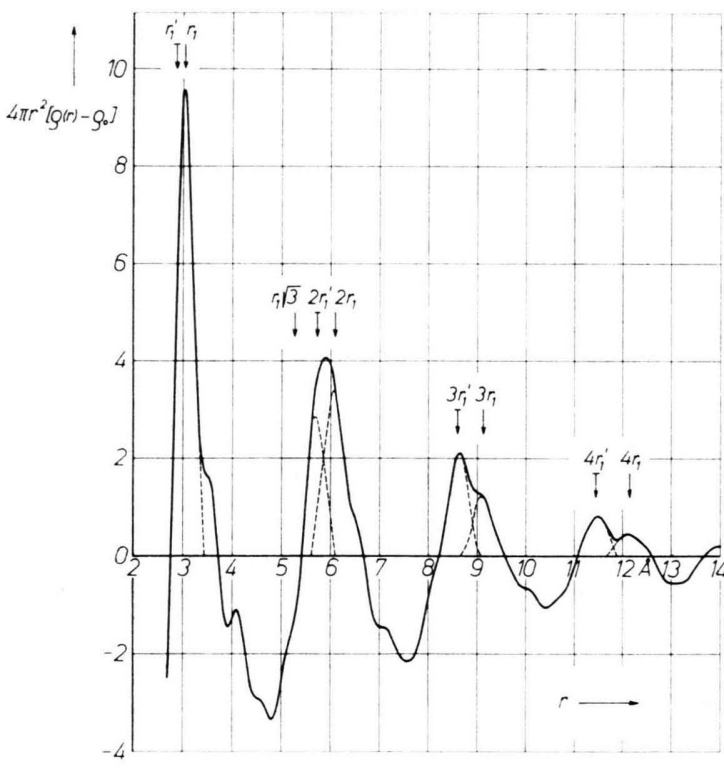

1 a

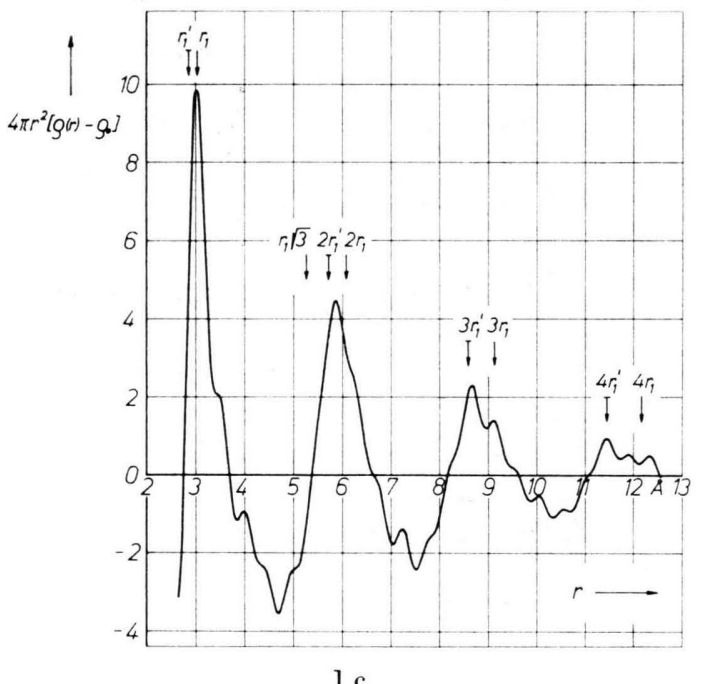

$1 \mathrm{c}$

tisch dieselben sind. Die Maxima sind also reell. Beim Übergang zu großer Integrationslänge $s_{2}$ erfaßt man auch den hinteren Teil der $i(s)_{\text {exp. }}$-Kurve und damit die äußeren Interferenzen der Flächengitter-Struktur, die hier stärker zur Geltung kommen. Mit wachsender Integrationslänge $s_{2}$ verschiebt sich daher das erste Maximum ${ }^{3 a}$ nach kleinen $\mathrm{Ab}$. standswerten. Das 4. Maximum der dichtesten Kugelpackung bei $r=4 r_{1}=12,16 \AA$ zeigt wegen des wach-

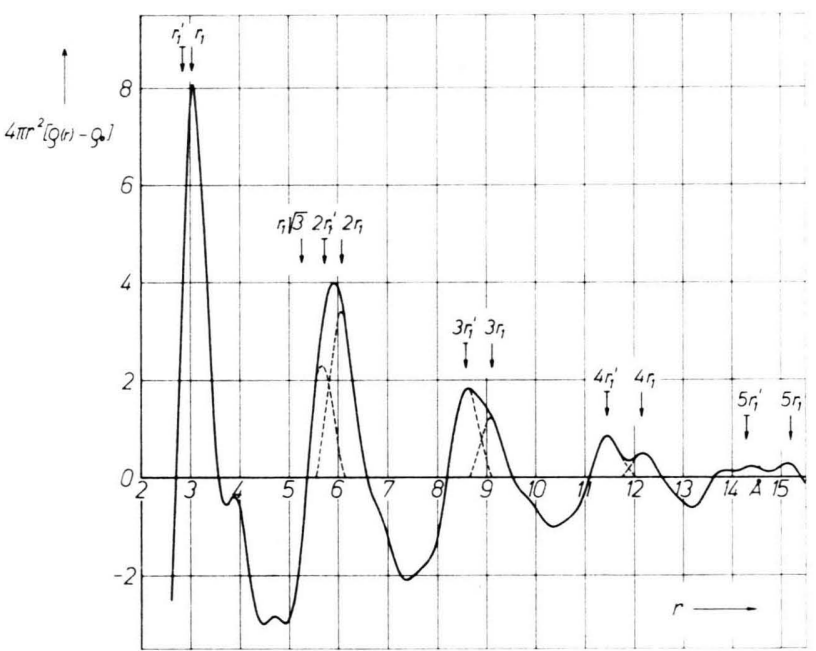

$1 \mathrm{~b}$

Abb. 1 a, b, c. $4 \pi r^{2}\left[\varrho(r)-\varrho_{0}\right]-$ Kurven von flüssigem Quecksilber.

$\downarrow=$ Berechnete Atomabstände der dichtesten Kugelpackung $r_{v}=v r_{1}$ mit $r_{1}=3,04 \AA$;

$\downarrow=$ Berechnete Atomabstände der Flächengitter-Struktur $r_{v^{\prime}}=v r_{1}^{\prime}$ mit $r_{1}^{\prime}=2,86 \AA$.

Abb. 1 a bei $s_{2}=12,6$, Abb. 1 b bei $s_{2}=8,4$ und Abb. $1 \mathrm{c}$ bei $s_{2}=13,5$ erhalten.

\begin{tabular}{ccrrrrc}
\hline 1 & \multicolumn{1}{c}{2} & \multicolumn{2}{c}{3} & \multicolumn{2}{c}{4} & 5 \\
\hline 3,05 & 5,93 & 8,64 & - & 11,44 & 12,17 & $s_{2}=8,4$ \\
3,03 & 5,87 & 8,62 & $\approx 9,09$ & 11,40 & 12,29 & $s_{2}=11,6$ \\
3,03 & 5,87 & 8,64 & $\approx 9,09$ & 11,47 & 12,11 & $s_{2}=12,6$ \\
3,01 & 5,86 & 8,65 & 9,09 & 11,44 & 12,33 & $s_{2}=13,5$
\end{tabular}

Tab. 1. Experimentelle Atomabstände in flüssigem $\mathrm{Hg}$ bei verschiedenen Integrationslängen $s_{2}$.

3: In gleicher Weise verschiebt sich nach Tab. 1 das zweite Maximum, nicht aber das dritte, da die zum dritten Maximum gehörigen Oszillationen der dreifachen Atomabstände $3 r_{1}$ und $3 r_{1}{ }^{\prime}$ für $s_{2}>8,4$ bereits abgeklungen sind. Weiter geht das Verhältnis der Atomzahlen $N_{\text {Kug. }}$ und $N_{\text {Flg. bei- }}$ der Strukturen für das 2. Maximum $\left(N_{\mathrm{Kug}} / N_{\mathrm{Flg}} \text {. }\right)_{s_{2}=8,4}$

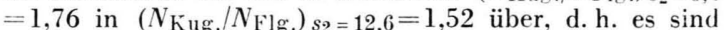
noch deutliche Oszillationen des doppelten Atomabstandes $2 r_{1}^{\prime}$ der Flächengitter-Struktur für $s_{2}>8,4$ vorhanden. Nach oben muß für das dritte Maximum das Verhältnis $N_{\text {Kug. }} / N_{\text {Flg. für }} s_{2}>8,4$ konstant sein. Man findet in den zwei betrachteten Fällen $N_{\text {Kug. }} / N_{\text {Flg. }}=0,63$ bzw. 0,58. 
senden Einflusses der Nebenoszillationen eine größere Lagestreuung (vgl. Abb. 1 b, wo der Einfluß der Nebenoszillationen gering ist). Wie bei Bi bildet sich der $r_{1} \sqrt{3}$-Abstand der dichtesten Kugelpackung in den Abb. $1 \mathrm{a}, \mathrm{b}$ und $\mathrm{c}$ nicht ab $\left(r_{1} \sqrt{3}=3,04 \sqrt{3} \AA\right.$ $=5,26 \AA)$.

Bei flüssigem $\mathrm{Hg}$ wurde der kürzeste Atomabstand der dichtesten Kugelpackung, abweichend vom Literaturwert $r_{12}=3,10 \AA$, zu $r_{1}=3,04 \AA$ gewählt, da anzunehmen ist, daß auch bei flüssigem $\mathrm{Hg}$ wie bei geschmolzenem $\mathrm{Bi}$ und $\mathrm{Ga}$ das erste Maximum vom kürzesten Atomabstand der dichtesten Kugelpackung festgelegt wird. Weiter zeichnet sich in Abb. 1 a auf der abfallenden Flanke des dritten Maximums, das vom dreifachen Atomabstand $3 r_{1}{ }^{\prime}$ der FlächengitterStruktur bestimmt wird, das Maximum des dreifachen Atomabstandes $3 r_{1}$ der dichtesten Kugelpakkung bei ${ }^{4} r=9,12 \AA=3 r_{1}$ ab $\left(r_{1}=3,04 \AA\right)$. Ferner tritt nach Tab. 1 das vierte Maximum der dichtesten Kugelpackung in zwei Fällen in der Nähe des vierfachen Atomabstandes $4 r_{1}=4 \cdot 3,04 \AA=12,16 \AA$ auf. Schließlich ist in Abb. $1 \mathrm{~b}$ sogar noch das fünfte Maximum der dichtesten Kugelpackung bei $r=15,14 \AA$ $\approx 5 r_{1}=5 \cdot 3,04 \AA=15,20 \AA \mathrm{zu}$ beobachten.

In flüssigem $\mathrm{Hg}$ liegen ähnliche Verhältnisse wie in geschmolzenem $\mathrm{Bi}$ vor. Bei geschmolzenem $\mathrm{Bi}$ treten nach Richter und Oenme ${ }^{5}$ (vgl. Abb. 1 a) das erste und zweite Maximum beim Pfeile $\downarrow$ der dichtesten Kugelpackung auf sowie das dritte und vierte Maximum zwischen den Pfeilen $\downarrow$ und $\downarrow$ beider Strukturen, das fünfte Maximum ist dagegen in zwei Maxima aufgespalten, und zwar in ein Maximum beim Pfeile $\bar{\downarrow}$ der Flächengitter-Struktur sowie in ein Maximum beim Pfeile $\downarrow$ der dichtesten Kugelpackung. Bei flüssigem $\mathrm{Hg}$ in Abb. 1 a tritt das erste Maximum beim Pfeile $\downarrow$ der dichtesten Kugelpak kung auf sowie das zweite Maximum zwischen den Pfeilen $\downarrow$ und $\downarrow$ beider Strukturen, deren Beiträge durch Aufteilung des zweiten Maximums eingezeichnet sind. Das dritte, vierte und fünfte Maximum sind dagegen in ein Maximum der FlächengitterStruktur $(\downarrow)$ und in ein Maximum der dichtesten

${ }^{4}$ Erst wenn man die Verschiebung dieses Maximums durch den steilen Abfall der Flanke berücksichtigt, findet man $r=9,12 \AA=3 r_{1}$ oder $r_{1}=3,04 \AA$ als kürzesten Atomabstand der dichtesten Kugelpackung (vgl. Tab. 1).

5 H. Richter u. H. Oehme, Z. Naturforschg. 22 a, 655 [1967].

6 R. Kaplow, S. L. Strong u. B. L. Averbach, Phys. Rev. 138, A 1336 [1965].

7 R. R. Fessler, R. Kaplow u. B. L. Averbach, z. Zt. im Druck.

${ }^{8}$ G. Breitling u. H. Richter, Z. Phys. 172, 338 [1963].
Kugelpackung $(\downarrow)$ aufgespalten, nicht zuletzt wegen der guten Homogenität der RöNTGEN-Strahlung. In flüssigem $\mathrm{Hg}$ liegen somit zwei Strukturen vor, die dichteste Kugelpackung und die Flächengitter-Struktur (vgl. dagegen Kaplow, Strong und Averbach ${ }^{6}$ sowie Fessler, Kaplow und Averbach ${ }^{7}$ ). Die Flächengitter-Struktur in flüssigem $\mathrm{Hg}$ hat im Gegensatz zum geschmolzenen $\mathrm{Bi}$ mit dem zugehörigen Gitter nicht den kürzesten Atomabstand gemeinsam. Bei $\mathrm{Hg}$ ist nämlich $\left[r_{1}\right]_{\mathrm{G}}=a_{\text {rhomboedr. }}=2,999 \AA$.

Als mittlere Atomzahlen für die geordneten Bereiche folgen aus den ersten drei Maxima der Abb. 1 a: $\quad N_{1,2,3}=\left(N_{\text {Kug. }}+N_{\text {Flg. }}\right)_{1,2,3} \approx 3,70 ; \quad 3,0$ und 1,5. Weiter erhält man durch Aufteilen des zweiten und dritten Maximums als Verhältnis der Atomzahlen beider Strukturen $\left(N_{\text {Kug. }} / N_{\text {Flg. }}\right)_{2 . \text { Max. }}$. $\approx 1,50$ und $\left(N_{\text {Kug. }} / N_{\text {Flg. }}\right)_{3 . \text { Max. }} \approx 0,60$. Danach ist für Abstandswerte $r \leqq 2 r_{1}$ die dichteste Kugelpakkung, für Abstandswerte $r \geqq 3 r_{1}{ }^{\prime}$ dagegen die Flächengitter-Struktur dominierend, wo die Abstandsschwankungen der Atome weniger groß sind. In dieser Hinsicht besteht Übereinstimmung mit den Ergebnissen der sukzessiven Analyse von Breituing und Richter ${ }^{8}$. Mit $\left(N_{\text {Kug. }}+N_{\text {Flg. }}\right)_{2 \text {. Max. }}=3,0$ und $\left(N_{\text {Kug. }} / N_{\text {Flg. }}\right)_{2 . \text { Max. }}=1,5$ findet man als Atomzahl der Flächengitter-Struktur $N_{\mathrm{Flg} .}=1,2$; das heißt, die Atomketten der Flächengitter-Struktur in flüssigem $\mathrm{Hg}$ sind bei linearer Ausdehnung an die 5 Atomabstände lang. Das gleiche Ergebnis erhält man aus dem dritten Maximum. Die Ketten können allerdings auch seitlich miteinander verknüpft sein. So kommen in flüssigem $\mathrm{Hg}$ die gerade Atomkette der dichtesten Kugelpackung und die gerade Atomkette der Flächengitter-Struktur - im letzten Falle vielleicht in Bündeln von Einzelketten - nebeneinander vor. Bei der geraden Atomkette der dichtesten Kugelpackung muß man eine flächenhafte oder eine räumlich dichteste Packung der Atome annehmen, sonst wäre der Atomabstand $r_{1}=3,04 \AA=r_{12}$ nicht möglich.

Bei geschmolzenem $\mathrm{Au}, \mathrm{Ag}, \mathrm{Pb}, \mathrm{Tl}$ sowie bei $\mathrm{Na}$ und Cs setzt bei großen Abstandswerten eine geringe Verkürzung ${ }^{9}$ der Atomabstände innerhalb der Zick-

${ }^{9}$ Es ist zu beachten, daß man bei den festen amorphen Stoffen und teilweise auch bei den Metallschmelzen zwischen zwei Vorstellungen streng zu unterscheiden hat: 1. Eine Abstandsverkürzung, sie berücksichtigt die Verkürzung der Abstände innerhalb der Atomkette und 2. eine Streuung der Atome um die Gleichgewichtslage, sie wird durch die Breite der Maxima in der Atomverteilungskurve gemessen. 
zackkette der dichtesten Kugelpackung ein. Die Abstandsverkürzung innerhalb der Zickzackkette ist bei festem amorphem As, Ge und Si besonders groß.

Als Beispiel für die Verkürzung der Atomabstände bei festen amorphen Stoffen sei das amorphe As betrachtet (bezüglich der Abstandsverkürzung bei amorphem Se vgl. Richter und Breitling ${ }^{10}$ ). In Abb. 2 ist die $4 \pi r\left[Q(r)-Q_{0}\right]$-Kurve von festem amorphem As nach Richter und Breituing ${ }^{11}$ wieder-

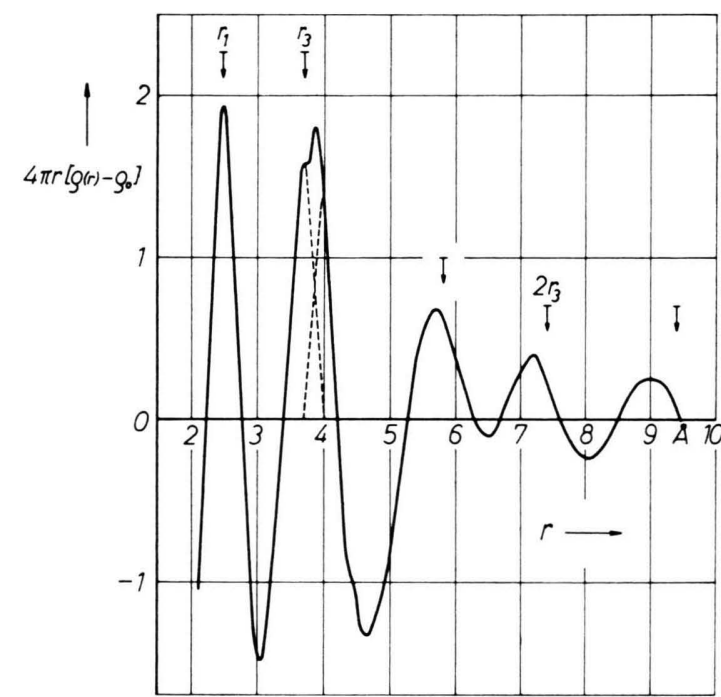

Abb. 2. $4 \pi r\left[\varrho(r)-\varrho_{0}\right]-$ Kurve von festem amorphem Arsen. $\downarrow=$ Berechnete Atomabstände innerhalb der ebenen Zickzackkette mit $r_{1}=2,51 \AA$ und $r_{3}=3,66 \AA$ als Abmessun. gen des Elementartetraeders.

gegeben. Der Grundbaustein von amorphem As ist wie im Gitter ein gleichschenkliges Tetraeder, seine Abmessungen sind durch die Abszissenwerte des ersten und zweiten Maximums der Atomverteilungskurve gegeben $\left(r_{1}=\left[r_{1}\right]_{\mathrm{G}}=2,51 \AA\right.$ und $r_{3}=3,66 \AA$ $\left.\neq\left[r_{3}\right]_{\mathrm{G}}=3,77 \AA\right)$. Mit ihrer Hilfe kann man die Atomabstände innerhalb der Tetraederkette berechnen. Das Experiment zeigt, daß sich bei amorphem As lediglich die ebene Zickzackkette abzeichnet, die von den Innenatomen der Tetraederkette gebildet wird. Auffallend ist in Abb. 2 die starke Abweichung (Abstandsverkürzung) der experimentellen Maximalagen von den berechneten Atomabständen innerhalb der ebenen Zickzackkette, deren Lagen durch die Pfeile mit Querbalken $\downarrow$ eingezeichnet sind. Die Ab-

10 H. Richter u. G. Breitling, Z. Naturforschg. 21 a, 1710 [1966].

11 H. Richter u. G. Breitling, Z. Naturforschg. 6 a, 721 [1951]; 13 a, 988 [1958]. - H. Richter u. G. Gommel, Z. Naturforschg. 12 a, 996 [1957]. weichung setzt hier bereits beim dritten Maximum ein; sie hängt offenbar mit der starken Auflockerung des festen amorphen As zusammen. Die Dichteänderung beim Ubergange: amorph $\rightarrow$ kristallin beträgt nämlich bei $A s \approx 36 \%$, bei Se dagegen nur $\approx 11 \%$.

Bei festem amorphem As treten starke Verkürzun. gen der Atomabstände innerhalb der Zickzackkette auf, sie sollten bei den Metallschmelzen nicht weniger groß sein. Überraschend ist daher die bei geschmolzenem $\mathrm{Au}, \mathrm{Ag}, \mathrm{Pb}$ und $\mathrm{Tl}$ sowie wie bei $\mathrm{Na}$ und Cs beobachtete und erst bei großen Abstandswerten einsetzende, geringe Verkürzung der Atomabstände innerhalb der Zickzackkette der dichtesten Kugelpackung (vgl. Richter und Breitling ${ }^{10,12}$ ), ebenso ist die bei festem amorphem und bei geschmolzenem $\mathrm{Bi}$ und $\mathrm{Ga}$ sowie bei flüssigem $\mathrm{Hg}$ fehlende Verkürzung der Atomabstände innerhalb der geraden Atomkette der dichtesten Kugelpackung auffallend. So erhielten Richter et al. ${ }^{13}$ bei festem amorphem $\mathrm{Bi}$ von $4{ }^{\circ} \mathrm{K}$ die Abstandswerte $r_{v}=\boldsymbol{v} r_{1}$ mit $r_{1}=3,32 \AA$ als kürzestem Atomabstand der dichtesten Kugelpackung. Weiter fanden Richter und Oenme $^{5}$ (vgl. Abb. 1 a) aus der Lage des ersten und zweiten Maximums der $4 \pi r^{2}\left[\varrho(r)-\varrho_{0}\right]$-Kurve einer Bi-Schmelze von $325^{\circ} \mathrm{C}$ die Abstandswerte $r_{1}=$ $3,32 \AA$ und $r=6,65 \AA \approx 2 r_{1}=6,64 \AA$ sowie bei Aufspaltung des fünften Maximums den Abstandswert $r=16,63 \AA \approx 5 r_{1}=16,60 \AA$. Danach beträgt der kürzeste Atomabstand der dichtesten Kugelpackung in einer Bi-Schmelze von $325{ }^{\circ} \mathrm{C}$ ebenfalls $r_{1}=$ $3,32 \AA$. Weiter treten die Atomabstände $r_{v}=v r_{1}$ wie bei festem amorphem Bi unverkürzt auf. Ähnlich liegen die Verhältnisse bei flüssigem $\mathrm{Hg}$. Hier findet man aus der Lage des ersten Maximums als kürzesten Atomabstand der dichtesten Kugelpackung $r_{1}=3,04 \AA$ und bei Aufspaltung des vierten und fünften Maximums die Abstandswerte $r=12,11 \AA$ $\approx 4 r_{1}=12,16 \AA$ und $r=15,14 \AA \approx 5 r_{1}=15,20 \AA$ (vgl. die Pfeile $\downarrow$ in den Abb. 1 a und b). Danach kommen auch in flüssigem $\mathrm{Hg}$ die Atomabstände $r_{v}=v r_{1}$ innerhalb der geraden Atomkette der dichtesten Kugelpackung unverkürzt vor.

Bei geschmolzenem $\mathrm{Bi}$, Sn und In sowie bei flüssigem $\mathrm{Hg}$ ist eine Verkürzung der Atomabstände $r_{v}{ }^{\prime}=v r_{1}{ }^{\prime}$ innerhalb der geraden Atomkette der Flä-

12 H. Richter u. G. Breitling, Adv. Physics 1967.

13 R. Leonhardt, H. Richter u. W. Rossteutscher, Z. Phys. 165, 121 [1961]. - H. Richter u. S. Steeb, Z. Metallkde. 50, 369 [1959]. - S. Stees, Dissertation, Techn. Hochschule Stuttgart 1958. 
chengitter-Struktur ebenfalls nicht festzustellen (bezüglich $\mathrm{Hg}$ vgl. die Pfeile $\downarrow$ in den Abb. 1 a und b). Auch hier ist die Übereinstimmung der beobachteten Atomabstände mit den berechneten Abstandswerten überraschend gut.
Die umfangreichen Fourier-Analysen wurden mittels des elektronischen Rechenautomaten (ER 56) wieder von Herrn Dipl.-Phys. R. Leonhardt durchgeführt. Wir danken ihm hierfür herzlich, ebenso danken wir der Deutschen Forschungsgemeinschaft für die vielseitige Unterstützung der vorliegenden Untersuchungen.

\title{
Nonlinear Incoherent Light Scattering
}

\author{
A. Salat \\ Institut für Plasmaphysik GmbH, Garching bei München \\ (Z. Naturforschg. 22 a, 662-670 [1967] ; received 3 February 1967)
}

\begin{abstract}
Classical equations are used to calculate nonlinear incoherent light scattered from two coherent beams of electromagnetic radiation of different frequencies propagating in a homogeneous plasma without magnetic field. With a suitable choice of the difference frequency, enhanced light scattering occurs near the plasma frequency. In this case the frequency spectrum is mainly given by the thermal ion density fluctuations.
\end{abstract}

Nonlinear processes, occurring when one or more electromagnetic waves propagate in a collisionless plasma, have been examined both theoretically ${ }^{1-8}$ and experimentally ${ }^{9}$. Theoretical work is of two kinds. In the first type, the plasma is treated using a collective model, be it the Vlasov equation or macroscopic equations. This produces nonlinear waves with frequencies, wave numbers and directions uniquely determined by the incoming (primary) waves ${ }^{4-9}$ because of energy and momentum conservation. (Though, as a result of refraction, a finite plasma volume makes a finite width of the angle of propagation.) In the second type ${ }^{1-3}$ the discrete plasma structure is considered explicitly, which leads to new effects. Each particle separately can exchange energy and momentum with the waves. Since, moreover, all particles interact via Coulomb forces, this presents an intricate problem which to the author's knowledge has been treated hitherto mainly in terms of quantum field theory with results of various authors that are not in agreement ${ }^{2}$. This

1 H. Cheng and Y. C. Lee, Phys. Rev. 142, 104 [1966].

2 G. Baym and R. W. Hellwarth, IEEE J. Quant. Electronics QE-1, 309 [1965].

3 P. M. Platzmann and N. Tzoar, Phys. Rev. 136, 11 [1964]. - D. F. DuBors and V. Gilinsky, Phys. Rev. 135, 995 [1964]. - H. L. Berk, Phys. Fluids 7, 917 [1964].

4 H.S. C. Wang and M.S. Lajko, Phys. Fluids 6. 1458 [1963].

5 W. H. KegeL, Z. Naturforschg. 20 a, 793 [1965]. - D. Montgomery, Physica 31, 789 [1965].

${ }^{6}$ N. M. Kroll, A. Ros, and N. Rostoker, Phys. Rev. Letters 13, 83 [1964]. - A. Salat, Z. Naturforschg. 20 a, 690 [1965]. work contains a purely classical description of nonlinear wave phenomena in which the particle aspects of the plasma are included, and the difficulties involved in quantum field theoretical representations are avoided.

In section 1 the problem is specified and the equations for handling it are given. Sections 2 and 3 give a brief summary of results relating to thermal plasmas and linear wave effects. In section 4 nonlinear effects are derived, which are discussed in sections 5 and 6 .

\section{Basic Equations}

The nonlinear interaction of electromagnetic waves will be specified here in the following way: Two electromagnetic waves having the frequencies $\omega_{\sigma}, \sigma=1,2$ the directions $\boldsymbol{n}_{\sigma}$ and arbitrary polarisation propagate in a homogeneous isotropic plasma: What are the nonlinear (quadratic) effects that can be seen outside the plasma?

7 R. F. Whitmer and E. B. Barret, Phys. Rev. 121, 661 [1961] ; 125, 1478 [1962]. - L. M. Gorbunov, V. V. Pustovalov, and V. P. Silin, Soviet Phys.-JETP 20, 967 [1965]. - A. Salat and A. Schlüter, Z. Naturforschg. 20 a, 458 [1965].

8 R. F. Whitmer, E. B. Barret, and S. J. Tetenbaum, Phys. Rev. 135, 369 [1964].

9 B. Blachier, J.-L. Delcroix, and E. Leiba, C. R. Acad. Sci. Paris 262, 472 [1966]. - R. F. Whitmer, E. B. Barret, and S. J. Tetenbaum, Phys. Rev. 135. 374 [1964]. 